\title{
Ultra-High Efficiency, High-Concentration PV System Based On Spectral Division Between GaInP/GaInAs/Ge And BPC Silicon Cells
}

\author{
P. Benítez ${ }^{1,2}$, R. Mohedano ${ }^{1}$, M. Buljan², J.C. Miñano ${ }^{1,2}$, Y. Sun ${ }^{1}$, W. Falicoff ${ }^{1}$, J. \\ Vilaplana $^{1}$, J. Chaves ${ }^{1}$, G. Biot ${ }^{2}$, J. López ${ }^{2}$ \\ ${ }^{1}$ LPI, +1 (626) 296-6461, pbenitez@lpi-europe.com, Altadena CA, US \\ ${ }^{2}$ Universidad Politécnica de Madrid (UPM) Cedint, Madrid, Spain
}

\begin{abstract}
A novel HCPV nonimaging concentrator concept with high concentration $(>500 \mathrm{x})$ is presented. It uses the combination of a commercial concentration GaInP/GaInAs/Ge 3J cell and a concentration Back-Point-Contact (BPC) concentration silicon cell for efficient spectral utilization, and external confinement techniques for recovering the 3J cell's reflection. The primary optical element (POE) is a flat Fresnel lens and the secondary optical element (SOE) is a free-form RXI-type concentrator with a band-pass filter embedded it, both POE and SOE performing Köhler integration to produce light homogenization. The band-pass filter sends the IR photons in the $900-1200 \mathrm{~nm}$ band to the silicon cell. Computer simulations predict that four-terminal terminal designs could achieve $\sim 46 \%$ added cell efficiencies using commercial 39\% 3J and 26\% Si cells. A first proof-of concept receiver prototype has been manufactured using a simpler optical architecture (with a lower concentration, $\sim 100 \mathrm{x}$ and lower simulated added efficiency), and experimental measurements have shown up to $39.8 \% 4 \mathrm{~J}$ receiver efficiency using a $3 \mathrm{~J}$ with peak efficiency of $36.9 \%$.
\end{abstract}

Keywords: High concentration, concentrator, spectrum splitting, Köhler integration.

PACS: 42.15.Eq, 42.79.Ek, 88.40.F, 88.40.H, 88.40.jj

\section{INTRODUCTION}

Presently, the high concentration PV industry (HCPV) is focused on commercializing products based on triple-junction (GaInP/GaInAs/Ge) solar cells, to take advantage of the high efficiency these cells provide. Commercial cells from a variety of suppliers are close to $40 \%$ average efficiency, not far from the present world record of GaInP/GaInAs/Ge technology, 41.6\% [1]. Even though competition has lowered triple-junction cell and assembly cost, these are still high enough to require high concentration $(>500)$ to be competitive in terrestrial applications.

For improving the utilization of the solar spectrum, one of the most promising strategies is the use of four or more p-n junctions. Different strategies have been followed to increase the number of junctions built into a solar cell (specially promising are inverted metamorphic cells, and wafer bonding/ layer transfer processes). An alternative approach consists of using separate cells and dichroic optical filters. Compared to single cell solutions, this approach has the possibility of avoiding the current-mismatch losses by multiple terminal configurations, and of eliminating the lattice matching prerequisites of monolithic growth. However, increased material cost and system complexity make the development of a commercially competitive product a challenging task. In the last few years, the dichroic beam-splitting approach is capturing renewed interest [2-6].

On the other hand, reflection losses of present $3 \mathrm{~J}$ cells are the main cause limiting their External Quantum Efficiency (EQE), and they are produced by the reflection on the metallic front gridlines and the Fresnel reflection on the semiconductor surface. This surface is no textured but AR coated, and the AR coating of present commercial cells is optimized for the $400-900 \mathrm{~nm}$ range to the $2-3 \%$ levels (since the Ge junction has excess of current), but the average reflection for the full in the further infrared is higher. Two different strategies have been used in the past to minimize the reflection of useful light. One consists in preventing the light from hitting the grid lines, for instance using refractive prismatic covers aligned over the grid lines (seminal work done by O'Neill, US Patent No. 4,711,972). This approach does not recover the light reflected on the semiconductor surface, needs very precise alignment, and is not effective under 
wide-angle illumination (as occurs in highconcentration systems), especially when a secondary optical element (SOE) is optically coupled to the cell On the contrary, a second strategy, referred to as External Confinement [8], is more compatible with high concentration. It consists on using a mirror cavity that collects the light reflected by the cell (either by the gridlines or by the semiconductor surface) and sending it back to the cell. The cavities do not need precise alignment, but have the drawback that they still lose the photons absorbed on the grid lines.

For present triple-junction cells, the light reflected on the semiconductor surface is specular (the cell surface roughness is very small, similar to good optical mirrors), while the light reflected on the grid lines has a significant diffuse component caused by the grid line geometry and roughness. However, it is remarkable that the roughness and imperfections of the grid line surface still keep the same cylindrical symmetry, as a good approximation (see FIGURE 1). Therefore, the direction of the scattered light conserves the vector component along the grid line, and a light ray is scattered inside the surface of a cone. This is important for the design of the concentrator and the external cavity (which traditionally has assumed random Lambertian scattering): the illumination of the cell need only be done from one hemisphere, while the cavity occupies the other (asymmetric cavity).
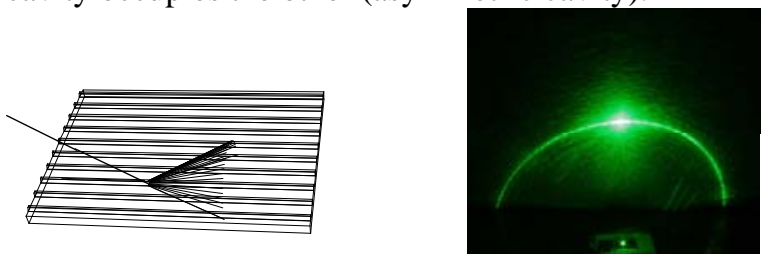

FIGURE 1. The irregularities and roughness of the gridlines of a commercial concentration triple-junction cell has approximately cylindrical symmetry, which allows efficient external confinement with an asymmetric cavity.

Here we present a novel HCPV nonimaging concentrator concept with high concentration $(>500 x)$ is presented. It uses the combination of a commercial concentration GaInP/GaInAs/Ge 3J cell and a BackPoint-Contact (BPC) concentration silicon cell for efficient spectral utilization, and external confinement techniques for recovering the $3 \mathrm{~J}$ cell's reflection. The silicon band-gap is nearly ideal for combining with the other three gaps (with a theoretical limit of $57 \%$ for two terminal devices [10]), which makes the combination very attractive. Moreover, the current densities that result of the idealized combination in four terminal operation are very adequate (see FIGURE 2): the silicon cell, whose full-spectrum current would be $44.4 \mathrm{~mA} / \mathrm{cm} 2$, gets reduced to a $27 \%$ level $(0.27=12.1 / 44.4)$, which implies that under equal geometrical concentration for both cells, if the $3 \mathrm{~J}$ cell is operated at its optimized 500x current gain, the silicon cell will then be run at $0.27 \times 500=135$, which close to its optimum too [7]. In the next sections, the features of our proposed solution will be described and non-idealized models presented, together with the first experimental results.

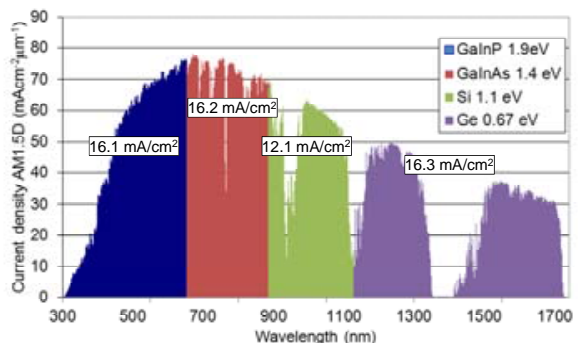

FIGURE 2. Band-gap calculation of maximum current densities (at 1-sun) of a four-terminal combination of a commercial concentration GaInP/GaInAs/Ge 3J cell and a Back-Point-Contact (BPC) concentration silicon cell.

\section{CONCENTRATOR DESIGN}

\section{Fresnel-RXI Köhler concentrator}

The concentrator concept [9] is described in FIGURE 3. The primary optical element (POE) is a flat FresnelKöhler lens and the secondary optical element (SOE), a free-form (i.e., with no rotational or linear symmetry) Köhler RXI-RR concentrator, with an embedded flat band-pass filter. The letters naming the device is the usual nomenclature of the SMS method [11], and refers to the type of surface $(\mathrm{R}=$ refraction, $\mathrm{X}=$ reflection, $\mathrm{I}=$ Total Internal Reflection) that reflects or refracts the light rays while crossing the device. In the RXI-RR concentrator, the RR illuminates the BPC silicon cell, while the RXI illuminates the triplejunction cell. This illumination is asymmetric from one hemisphere, allowing the use of an external confining cavity in the other hemisphere to efficiently collect the light reflected by the grid lines and the semiconductor surface of the triple junction cell.

The Fresnel-Köhler families for HCPV (named FK, XRK, XXRK, F-RXI) have been introduced by LPI in the recent years [12-15], as novel highperformance concentrators that provide not only high concentration and high acceptance angles (i.e., high CAP), but also excellent uniformity of illumination on the square cell without the effects of chromatic aberration on the cell. Particularly, the free-form RXI has achieved the highest CAP reported to date for refractive POE's [15]. This high "CAP budget" is important in our case, as it allows us to reduce it in exchange for leaving angular space for the external cavity, and still achieving the necessary high concentration and acceptance angle. 


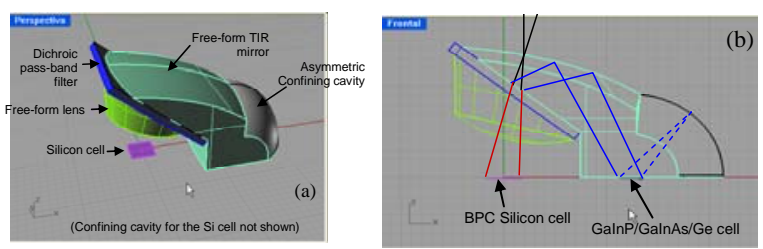

FIGURE 3. Köhler RXI-RR SOE concentrator, with the flat band-pass filter and the $3 \mathrm{~J}$ and BPC silicon cells.

There are several additional practical aspects of our RXI-RR design that help to reduce the added complexity of the beam splitting systems. First, the filter, the SOE for the BPC silicon cell (i.e, the RR) and the SOE for the triple-junction cell (i.e, the RXI) form a single piece of dielectric, which simplifies its mounting. Secondly, the two cells are located on the same plane, simplifying the heat management and wiring. Thirdly, there are no optical surfaces in contact with the cell rim, which differs from other traditional HCPV secondary optics (such as prism homogenizers). This makes the encapsulation of the cells much easier and more robust, since there is no threat of light loss due to meniscus effects by the silicone rubber coupler), and therefore more suitable for high-yield in mass production [13].

Alternative SOE's architectures have been also considered. One particularly interesting is the freeform RXI-RI ${ }^{2}$ configuration, which allows the Silicon and $3 \mathrm{~J}$ cell to be located not only coplanar but also closer than in the RXI-RR, so they can share the receiver substrate. This design, with a geometrical concentration of $625 \mathrm{x}$ for the $3 \mathrm{~J}$ cell and 560x for the Si cell, shows a modeled optical efficiency of $85 \%$ for both the MJ and silicon cells (no AR coating considered) and a well-balanced acceptance angle of $\pm 0.9^{\circ}$ for both cells.

\section{Band-pass filter}

The band-pass filter in the RXI-RR SOE is design to minimize its cost of the band-pass filter and guarantee its performance. For that purpose, first we have restricted our concentrator design to operate with a flat filter. Then, even having dozens of layers, the filter can be manufactured cost-effectively in large flat plates which are diced afterwards. Still, the filter will need to work under concentration $(>40-50 x)$, to make its cost affordable.

Second, the band-pass filter is designed to match the currents of the bottom $(\mathrm{Ge})$ junction to the top (GaInP) and the middle (GaInAs) junctions, minimizing the losses not considered in the ideal 57\% mentioned in FIGURE 2 (that ideal calculation assumes pill-box type spectral splitting and full absorption, and none can be achieved in practice). For instance, the External Quantum Efficiency (EQE) of BPC silicon cell is not close to $100 \%$ in the $950-1200$ $\mathrm{nm}$ (due to the indirect band gap of the silicon), as shown in ref [7]. On the other hand, in a real filter the high slopes of the edges of the transmission band are only obtained for moderate values of the average angle of incidence on the filter, $\beta_{F}$, and the angular radius of that light bundle on the filter, $\alpha_{F}$. Since the quantity $\left(\sin \alpha_{F} \cos \beta_{F}\right)^{2}$ in inversely proportion to the concentration on the filter, the need of moderate values of $\alpha_{F}$ suggests to limit the concentration on the filter to the minimum that is cost effective ( 40-50x). Therefore, the SOE, after the filtering, has to provide the additional 10-12x concentration on the cells. We have selected $\beta_{F}>25^{\circ}$ and $\alpha_{F}>5^{\circ}$, when the surrounding media is a dielectric material $(n \approx 1.5)$.

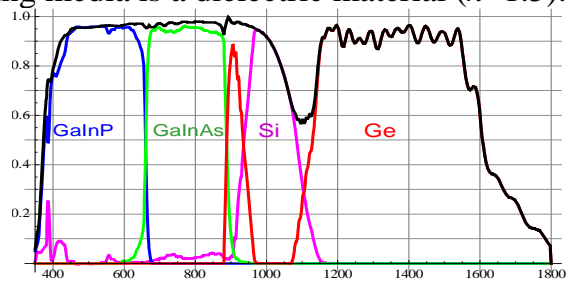

FIGURE 4. EQE of the four junctions of an RXI-RR design for four terminals, and its aggregation. Our modeling indicates that this design should achieve about $46 \%$ efficient under AM1.5d with 39\% 3J cell and 26\% silicon cell.

\section{PROOF-OF-CONCEPT PROTOTYPE}

The proof-of-Concept prototype has been manufactured by LPI and UPM-Cedint based on a simpler optical concept (see Fig 2). It is composed of an RXI prism, a 41-layer stop-band dichroic filter, a GaInP/GaInAs/Ge cell and a BPC concentration silicon cell. As opposed to the proposed RXI-RR system, this prototype does not use a confinement cavity, but sends the light reflected by the stop-band filter to the silicon cell. This lowers the expected efficiency in 4-terminal operation due to the loss of the reflected photons over $1200 \mathrm{~nm}$ (previously used by the Ge junction) and due to lower voltage at which the carriers generated by reflected photons below $900 \mathrm{~nm}$ will be extracted. However, the prototype is expected to illustrate experimentally the high potential of this spectrum splitting concept.

Optical transmission measurements of the manufactured and the filter coating has shown important deviations from the design values, predicting a higher reflection of photons below 900nm to the silicon cell. Also, the measurement of the manufactured AR coating show that it produces a transmission $\sim 96 \%$ over $900 \mathrm{~nm}$, instead of the designed 98\%.

Regarding the electrical measurement, two 3J cells were selected and measured with a standard flash 
tester at $50 \mathrm{~W} / \mathrm{cm}^{2}$ and AM1.5d spectrum, and they showed $39.4 \%$ and $39.5 \%$ efficiencies. One of them was then encapsulated with a silicone rubber as optical coupler to the RXI prism with a BPC silicon cell. Later, measurements under real sun on March $21^{\text {st }}$ in Madrid, using a concentrator test rig that was developed to produce a well-defined square irradiance distribution of $6.5 \times 6.5 \mathrm{~mm}^{2}$ with $7.33 \mathrm{~W}( \pm 2 \%)$ at @900W/ $/ \mathrm{m}^{2}$, to be fully inscribed in the receivers to measure, producing $\sim 10 \mathrm{~W} / \mathrm{cm}^{2}$ average concentration on the $3 \mathrm{~J}$ cell area. Under that concentrator test rig, the reference $3 \mathrm{~J}$ cell showed a peak $36.8 \%$ efficiency (36.9\% corrected for Tcell $=25^{\circ} \mathrm{C}$ ), lower than the one at the simulator due to the lower concentration set up. On the other hand, the $4 \mathrm{~J}$ RXI prism receiver assembly gave $39.8 \%$ with $4 \mathrm{~T}$ ( $39.7 \%$ corrected for Tcell $\left.=25^{\circ} \mathrm{C}\right)$, and $39.7 \%$ with $2 \mathrm{~T}$. Then, output peak-power gain of the $4 \mathrm{~J}$ over the $3 \mathrm{~J}$ was about $8 \%$, which is very significant and promising, especially with the problems found in the coatings made in this firstiteration (confirmed by the higher current measured on the silicon cell, with respect to the expected values; see FIGURE 6), and encouraged to improved them to achieve the $13 \%$ predicted by our model.
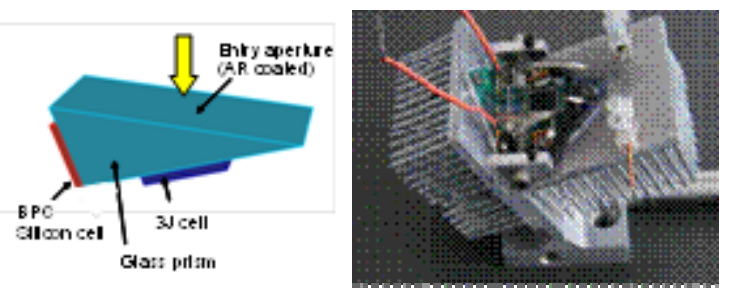

FIGURE 5. Proof-of concept prototype 4J module

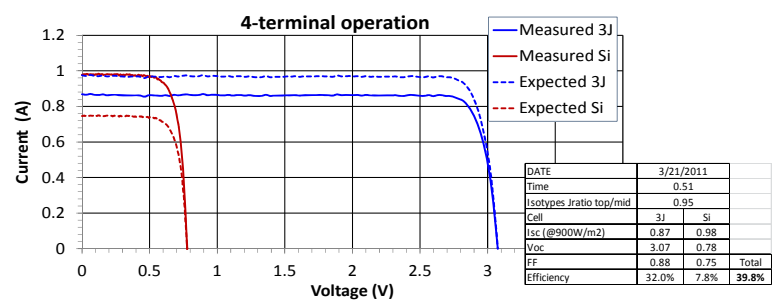

FIGURE 6. Peak-efficiency I-V curves (measured and expected) of the $4 \mathrm{~J}$ receiver module.

\section{CONCLUSIONS}

Novel high-concentration Köhler SOE concepts with integrated dichroic flat filter for coplanar commercial 3J cell and BPC Si cells, with expected equivalent cell efficiency of $46 \%$ from $39 \% 3 \mathrm{~J}$ cells ( $+17 \%$ gain). First simpler prototype shows a $+8 \%$ efficiency gain, and next iteration with improved filter targets a $+13 \%$ gain and $43 \%$ efficiency at $300 x$. Future efficiency over $50 \%$ could be attainable with custom designs $3 \mathrm{~J}$ (with better Ge sub-cells) and Si cells (with better IR response).

TABLE 2. Measured efficiencies of the $3 \mathrm{~J}$ reference cell and $4 \mathrm{~J}$ prism receiver in $4 \mathrm{~T}$ operation (corrected for Tcells $=25^{\circ} \mathrm{C}$ ).

\begin{tabular}{lcc}
\hline & Measured & Expected \\
\hline Reference 3J & $36.9 \%$ & $37.4 \%$ \\
4J prism (4T) & $39.7 \%$ & $42.5 \%$ \\
Gain & $+8 \%$ & $+13 \%$ \\
\hline
\end{tabular}

\section{ACKNOWLEDGMENTS}

The authors thank V. Garbousian, A. Plesniak and G. Kinsey, from Amonix Inc. for their support for this work. The authors also thank G. Sala, R. Herrero and R. Nuñez, from IES-UPM, for their help to characterize the $3 \mathrm{~J}$ cells outdoors and indoors.

\section{REFERENCES}

1. M.A. Green, et al. "Solar cell efficiency tables”. Prog. Photovolt: Res. Appl. 2009; 17(5): 320-326.

2. A. Barnett et al., "Very High Efficiency Solar Cell Modules”, Prog. Photovolt: Res. Appl. 2009; 17:75-83.

3. M. A. Green, A. Ho-Baillie. "Forty three per cent composite split-spectrum concentrator solar cell efficiency”, Prog. Photovolt: Res. Appl. 2010; 18:42-47

4. L.M. Fraas, et al. "Toward $40 \%$ and Higher Multijunction Cells in a New Cassegrain PV Module”, 31st IEEE PVSC, Florida, USA (Jan. 2005).

5. E. Shifman, US pat. application 2008/0000516.

6. B. Mitchell et al. "Four-junction spectral beam-splitting photovoltaic receiver with high optical efficiency” Prog. Photovolt: Res. Appl., Online, july, (2010).

7. A. Slade et al., "Issues encountered in the manufacture of $125 \mathrm{~mm}$ thick, 26\% efficient silicon solar cells in microelectronics foundries”, 19th EU PVSEC, (2004).

8. J. C. Miñano, "Optical confinement in photovoltaics,“ in Physical Limitations to the Photovoltaic Solar Energy Conversion, A. Luque and G. L. Araújo, eds. (Hilger, Bristol, UK,1990).

9. US and International Patents Pending including: $P$, Benítez et al., "Photovoltaic Device” PCT publication WO 2010/016930 February 2, (2010).

10. R.R. King et al. "Progress in High-Efficiency III-V Multijunction Concentrator Solar Cells”, DOE Solar Program Review Meeting, Denver, April 16-19, (2007).

11. R. Winston, J.C. Miñano, P. Benítez, "Noinimaging Optics” (Academic Press-Elsevier, 2004).

12. P. Zamora, et al, "Advanced PV Concentrators", 34th IEEE PVSC, Philadelphia, (2009)

13. P. Benitez et al. "High performance Fresnel-based photovoltaic concentrator," Opt. Express 18, (2010) .

14. M. Buljan et al., "Improving performances of Fresnel CPV system: Fresnel-RXI Köhler concentrator”, 25th EU PVSEC, Valencia (2010).

15. A. Cvetkovic et al. "Characterization of Fresnel-Köhler Concentrator”, 25th EU PVSEC, Valencia (2010). 